\title{
Comparison of preparation techniques for nuclear materials for transmission electron microscopy (TEM)
}

\author{
A. Aitkaliyeva ${ }^{1}$, J. W. Madden, B. D. Miller, J. I. Cole, J. Gan \\ Idaho National Laboratory, Idaho Falls, Idaho, 83415
}

\section{Keywords}

Nuclear materials, electro-polishing, focused ion beam (FIB), precision ion polishing system (PIPS-2), transmission electron microscopy (TEM).

\begin{abstract}
Preparation of highly radioactive and irradiated nuclear fuels and materials for transmission electron microscopy (TEM) is accompanied with a set of unique challenges. The paper evaluates three specimen preparation techniques for preparation of irradiated materials and determines which technique yields to the most reliable characterization of radiation damage microstructure. Various specimen preparation artifacts associated with each technique are considered and ways of minimizing these artifacts are addressed.
\end{abstract}

\section{Introduction}

Exposure to radiation in nuclear reactor environment results in formation of diverse defect microstructures. Depending on irradiation temperature, neutron flux, and neutron fluence, neutron irradiation can produce various defects in materials: point defects, line defects (dislocation lines) [1], planar defects (dislocation loops) [2], and volume defects (voids, bubbles, and stacking fault tetrahedra) [3-4]. These defects can alter mechanical properties of the materials, lead to embrittlement of reactor structural materials during service life, and accelerate material failure. A firm understanding of radiation effects in reactor materials is imperative to understanding in-reactor degradation behavior, account for irradiation effects in design, and create new generation radiation-tolerant materials. Transmission electron microscopy (TEM) is a powerful tool for investigation of defect microstructures and obtaining atomic scale understanding of the radiation effects in nuclear fuels and materials. Therefore, detailed chemical and structural TEM characterization of radiation-induced changes in reactor material at the nano and atomic scale is of profound consequence for evaluation of the behavior of nuclear fuels and materials under irradiation.

Highly radioactive samples impose unique challenges when it comes to sample preparation for TEM analysis. For example, conventional sample preparation techniques, such as electro-polishing and ion milling, frequently require close-in, hands-on manipulation of the

${ }^{1}$ Corresponding author

Idaho National Laboratory, P.O. Box 1625, M.S. 6188, Idaho Falls, ID 83415-6188, assel.aitkaliyeva@inl.gov, T: 208-533-8853, F: 208-533-7863. 
sample for extended periods of time. In the past few years, focused ion beam (FIB) technology has gained popularity in nuclear research community since it allows characterization of highly radioactive nuclear fuels and materials with minimal external hands-on sample processing and minimal personnel radiation exposure. One of the main advantages of the FIB in nuclear materials' research is the reduction of the specimen mass from the bulk to TEM lamella within confines of the FIB chamber, which reduces the radioactivity of the final product by multiple orders of magnitude. However, preparation of specimens for TEM analysis in FIB can lead to formation of highly undesirable sample preparation artifacts such as ion beam-induced defects and amorphization. Energetic ion beams produce atomic displacements and point defects in the material. The damage accumulated during sample preparation involving ion milling can look very similar (depending on irradiation conditions) to the damage produced in nuclear materials irradiated to low displacements per atom (dpa) levels and/or lower temperatures. Hence, one has to consider suitability of various techniques in preparation of irradiated materials, and determine which technique will yield a most reliable characterization of radiation damage microstructure. In the present contribution, we evaluate appropriateness of different sample preparation techniques in the field of nuclear materials. We choose to examine HT-9, Fe, Fe-12Cr, and Al specimens because they are either considered for application in a reactor environment, currently being used as cladding material, and/or are integral components of a variety of steels used in nuclear field.

\section{Materials and methods}

A variety of nuclear materials have been selected for this study. Alloy HT-9 has been included since it is one of the ferritic-martensitic alloys that are considered to be candidate cladding and structural alloys for application in fission, fusion, and fast reactors. Alloys based on Fe-Cr system are considered for reactor fuel cladding and structural material applications for several types of next generation reactors. Hence, $\mathrm{Fe}$ and $\mathrm{Fe}-12 \mathrm{Cr}$ specimens were selected to provide a basis for evaluation of radiation performance of the more complex alloys. Aluminum specimens were included because $\mathrm{Al}$ alloys are favorable structural and cladding materials due to their good corrosion resistance, high thermal conductivity, ductility, toughness, and a low neutron absorption cross-section [5].

Specimens were prepared for defect and sample preparation artifact analysis using both conventional means such as electro-polishing and ion milling, FIB-based lift-out technique, and combination of FIB with post-processing step in precision ion polishing system (PIPS). All selected specimens were mechanically polished to a mirror surface using $1 \mu \mathrm{m}$ diamond solution. Specimens for conventional sample preparation procedure were initially polished to the thickness of $\sim 120 \mu \mathrm{m}$. Plain view specimens for TEM examination were prepared by electropolishing at temperatures below $-40^{\circ} \mathrm{C}$ in a solution of 5\% perchloric acid and $95 \%$ methanol in a SouthBay Model 550D single vertical jet electro-polisher. Cross-sectional TEM specimens were prepared in a FEI Quanta 3D field emission gun (FEG) dual beam scanning electron microscope/focused ion beam (SEM/FIB) system using an in-situ lift-out technique. All 
specimens were lifted-out and thinned at $30 \mathrm{keV}$ and then carefully cleaned at lower energies (5 $\mathrm{keV}$ and $2 \mathrm{keV}$ ) and low beam currents (not exceeding $48 \mathrm{pA}$ at $5 \mathrm{keV}$ and not exceeding $16 \mathrm{pA}$ at $2 \mathrm{keV}$ ) to minimize $\mathrm{Ga}$ ion beam damage. A Gatan precision ion polishing system-2 (PIPS-2) was implemented in preparation of plain-view specimens and post-processing of FIB-prepared cross-sectional specimens. FIB-prepared cross-sectional specimens were milled in PIPS-2 at energies ranging from 2.0 to $0.1 \mathrm{keV}$ at shallow milling angles $\left(5^{\circ}\right)$. Quality of the prepared specimens was established through examination in a JEOL JEM 2010 transmission electron microscope operating at an accelerating voltage of $200 \mathrm{kV}$.

\section{Results and discussion}

Electro-polishing is considered to be a relatively reliable and reproducible sample preparation techniques for metals, which has a limited number of sample preparation artifacts associated with it [6]. The primary artifacts commonly formed include differential etching, deposition of debris of polished material due to inadequate rinsing, formation of surface films, pitting, and formation of hydrides in some susceptible materials, such as Zircaloy-4. Since this technique involves the usage of electrolytic solution and doesn't introduce any defects that can be confused with irradiation-induced defects, it has traditionally been preferred sample preparation method for irradiated materials. Over the past several years, the popularity of electropolishing began to diminish and FIB-based lift-out technique became preferable preparation techniques for irradiated materials. This was in part associated with the ability to produce sitespecific cross-sectional lift-outs in FIB, reduction of radioactivity of the prepared lamella that allowed acquisition of high-quality energy dispersive spectroscopy (EDS) data with reduced background intensity, and preservation of integrity of bulk samples and their availability for additional analysis, if needed, which is explained by the small size $(15 \mu \mathrm{m} \times 10 \mu \mathrm{m} \times 100 \mathrm{~nm})$ of FIB lift-outs. Figure 1 compares microstructure of unirradiated HT-9 specimen prepared for TEM analysis using a) electro-polishing and b) FIB-based lift-out technique. Both techniques yield good quality specimens without any notable sample preparation artifacts in case of HT-9, which suggests that this material is an acceptable candidate for post-irradiation examination in FIB and then TEM.

Despite its advantages, FIB has a major disadvantage when it comes to nuclear materials characterization - beam damage. It is well known that material removal in the FIB is achieved via milling/sputtering with gallium $(\mathrm{Ga})$ ion beam, and as a result a number of defects can be formed in the target material: vacancies, interstitials, and defect clusters. When the number of accumulated defects or the critical amount of deposited energy exceeds a threshold value, material can undergo a crystalline-to-amorphous transition. FIB-based sample preparation can also result in: formation of intermetallic phases and Ga precipitates [7], crystal size refinement [8], reorientation of crystallographic direction in some metals [9], curtaining of the irradiated fuel due to fission-gas-induced porosity [10], and differential thinning of diverse phase constituents in multi-component systems [11]. Lower accelerating voltages are known to produce a shallower amorphous layer and thus minimize FIB damage. Therefore a low-energy cleaning 
step is imperative in preparation of high-quality samples with minimal surface artifacts. Nonetheless, reducing the energy to $5 \mathrm{KeV}$ or even $2 \mathrm{keV}$ is not sufficient to eliminate defects produced in the specimen during sample preparation. Figure 2 shows microstructure of unirradiated pure Fe specimen prepared for TEM analysis using: a) electro-polishing and b) FIBbased lift-out technique. The microstructure of the electro-polished Fe specimen does not contain any surface artifacts or preparation-induced defects. The microstructure of unirradiated Fe prepared using FIB, on the other hand, contains extensive Ga ion beam damage even after careful cleaning at $2 \mathrm{keV}$.

The extent of sample preparation-induced damage can interfere with specimen analysis and in case of nuclear materials preclude accurate defect microstructure characterization. As it was mentioned earlier, damage accumulated during FIB-based sample preparation can look similar to the damage produced in nuclear materials irradiated to low displacements per atom (dpa) levels and/or lower temperatures. Figure 3 shows microstructure of a) unirradiated $\mathrm{Fe}$ specimen prepared using FIB tool and for comparison Fe specimens irradiated to b) $0.1 \mathrm{dpa}$ at $300^{\circ} \mathrm{C}$ with neutrons [12] and c) $0.5 \mathrm{dpa}$ at room temperature with $500 \mathrm{keV} \mathrm{Fe}$ ions [13]. All irradiated specimens shown in this paper have been prepared for TEM analysis using electropolishing method to avoid introduction of $\mathrm{Ga} / \mathrm{Ar}$ ion beam artifacts. TEM examination of irradiated Fe specimens confirmed the presence of defect clusters, dislocation lines, and small dislocation loops [12]. As it can be seen from the figure, preparation of such specimens using FIB tool would hinder rigorous irradiation-induced defect analysis.

Another comparison between FIB-produced defects and neutron/ion irradiation-induced defects is provided in Fig. 4. The microstructure of unirradiated Fe-12Cr specimen prepared using FIB-based lift-out technique is shown in Fig. 4(a), and microstructures of $12 \mathrm{Cr}$ steel irradiated with $1 \mathrm{MeV} \mathrm{Kr}$ ions at $200^{\circ} \mathrm{C}$ to $1 \mathrm{dpa}$ [14] and $\mathrm{Fe}-15 \mathrm{Cr}$ steel irradiated with neutrons to $2.8 \times 10^{22} \mathrm{n} / \mathrm{cm}^{2}$ at $450^{\circ} \mathrm{C}$ [15] are shown in b) and c), respectively. Small defect clusters, dislocation loops and lines, and helical dislocation configurations were reported in specimen irradiated with $\mathrm{Kr}$ ions to $1 \mathrm{dpa}$ [14], while dislocation loops arrays, dislocation networks, defect clusters, and voids were reported in specimens irradiated to $2.8 \times 10^{22} \mathrm{n} / \mathrm{cm}^{2}$ with neutrons [15]. Unirradiated FIB-prepared Fe-12Cr specimen contains a large number of defects and defect clusters despite prolonged cleaning at lower accelerating voltages at the final stages of specimen preparation.

As has been illustrated, Ga ion beam damage can be extensive enough to interfere with analysis of irradiated specimens and in some cases, depending on irradiation fluence and temperature, can be indistinguishable from neutron irradiation damage. These formed damaged layers, consisting of both amorphous material and implanted $\mathrm{Ga}$ ions, should be eliminated in order to perform an in-depth examination of material microstructure after irradiation. To reduce or completely eliminate sample preparation artifacts, energy of the impinging particles should be reduced to below $2 \mathrm{keV}$. However, only the most advanced FIB systems are capable of reducing ion energy below $2 \mathrm{keV}$ and these systems are not optimal for preparation of highly activated materials due to shorter working distances of $2 \mathrm{~mm}$ (as opposed to $10 \mathrm{~mm}$ in FEI Quanta 
microscopes). Therefore, post-processing of the FIB lamella has to be considered to minimize or eliminate this damage and ensure high quality analysis results.

Post-FIB processing of cross-sectional lamella can be done using a variety of advanced ion milling systems, in which energy of the beam can be reduced to $<500 \mathrm{eV}$. In this study, we implemented Gatan PIPS-2 system for final preparation of thin, high-quality FIB lift-out specimens for TEM analysis. However, other ion milling systems, such as Fischione NanoMill, can be used to eliminate FIB damage in materials. Lift-out specimens were initially prepared and thinned at $30 \mathrm{kV}$ to $\sim 300 \mathrm{~nm}$ in FIB system and then further thinned in PIPS-2. Note that if a different system, such as Fischione NanoMill, is used to eliminate FIB damage, the thickness of lamella has to be reduced to $100-120 \mathrm{~nm}$ in the FIB prior to post-processing. Unlike FIB, PIPS-2 system uses energetic Ar ions, not $\mathrm{Ga}$, to sputter target material and can reduce ion energy down to $100 \mathrm{eV}$. The thinning rate was controlled by careful selection of beam energy, current, and angle of incidence. Higher beam energies and angles of incidence are initially used to thin the specimen rapidly, and lower beam energies and angles of incidence are employed to remove damaged layers and clean the bulk specimen. Since PIPS-2 system is based on the usage of an ion beam, it can produce a variety of artifacts such as preferential milling, re-deposition of the sputtered material onto the specimen, localized heating of the specimen, and crystalline-toamorphous transition (amorphization) of the specimen due to bombardment with energetic ions. Even so, reduction of ion energy and milling angles in bulk specimen milling minimizes formation of ion beam damage and specimen heating, and low angles of incidence facilitate uniform thinning of dissimilar materials.

Figure 5 illustrates advantages of adding post-processing to the FIB preparation procedure by providing comparison of $\mathrm{Fe}-12 \mathrm{Cr}$ specimen microstructure (a) after electropolishing, (b) FIB-based lift-out, and (c) a combination of FIB-based lift-out technique with post-processing in PIPS-2. In the present work diffracting vectors and bright field microscopy were used to image defects in all analyzed specimens. Specimens were continuously tilted to identify all two-beam conditions existing in the specimen and to ensure thorough characterization of defects. The microstructures shown in figures 1-6 are representative of their corresponding specimens. TEM examination of the microstructure of electro-polished and postprocessed specimens did not reveal the presence of sample preparation artifacts and defects. TEM analysis of the FIB-prepared lamella, on the other hand, exposed a variety of Ga ion beaminduced defects, including point defects and defect clusters. Different contrast and lines going across micrographs depict thickness variations in the specimen.

Table 1 summarizes PIPS-2 parameters, which were selected in preparation of this specimen. The specimen was initially thinned in FIB system at $30 \mathrm{kV}$ to the thickness of $220 \mathrm{~nm}$ and then post-processed in PIPS-2 unit to the thickness of $50 \mathrm{~nm}$. As it has been mentioned previously, reduction of milling angles for bulk specimens aids in minimization of ion beam damage. However, it is advisable to use higher angles of incidence for FIB lamella (8-10 degrees). In our experience, lower milling angles can result in re-deposition of $\mathrm{Cu}$ or Mo from the grid onto specimen surface. Despite higher milling angles, lower beam energies implemented 
in milling of FIB lamella are sufficient to minimize beam damage. Liquid nitrogen cooling is typically used for temperature sensitive samples but in case of nuclear materials discussed in this paper liquid nitrogen and room temperature milling yielded similar results. Figure 5 shows that reducing ion energy from $2 \mathrm{keV}$ to $300 \mathrm{eV}$ allows preparation of high-quality specimens without any ion beam-induced artifacts. This is extremely important in microstructure and defect analysis of nuclear fuels and materials in which differentiation between various defects is of high importance.

Additional advantage of combining these two techniques is reduction of specimen preparation time. FIB based lift-out procedure on average can be completed within a few hours' time, depending on the material of interest and experience of user. By thinning the specimen not to electron transparency but to the thicknesses ranging from 200-400 nm, one can noticeably reduce sample preparation time in FIB. Thinning of such lamella in PIPS-2 system, in case of nuclear materials with average lamella size of $15 \mu \mathrm{m}$ in width and $10 \mu \mathrm{m}$ in height, requires on average 20-40 min. The milling rate depends on a variety of parameters, such as ion beam energy, angle of incidence, and material sputtering rate, and the provided estimate should be considered as a baseline and not an actual number valid for all types of nuclear materials. For example, post-processing of $\mathrm{Fe}-12 \mathrm{Cr}$ specimen shown in Fig. 5(c) was accomplished in $20 \mathrm{~min}$ in PIPS-2 and thickness of the lamella was reduced from $\sim 220 \mathrm{~nm}$ (after FIB) to $\sim 50 \mathrm{~nm}$ (after post-processing). PIPS-2 system, or any other system with comparable characteristics, can be also used to remove damage from specimens prepared using conventional methods such as electro-polishing. Electro-polished specimens sometimes have re-deposited debris of polished material and exhibit differential etching. Preferentially thinned electro-polished specimens not suitable for TEM characterization can be further thinned in PIPS-2 system, which will expose a damage-free area appropriate for TEM examination improving utilization of valuable irradiated specimens.

Figure 6 shows bright-field TEM micrographs of unirradiated Al specimen prepared using a) FIB-based lift-out and b) combination of FIB-based lift-out and post-processing in PIPS-2. Despite cleaning at $2 \mathrm{keV}$ to minimize Ga ion beam damage, a large number of ion beam-induced defects were found in $\mathrm{Al}$ specimen, as it can be seen in Fig. 6(a). An added postprocessing in PIPS-2 eliminated majority of Ga ion beam damage and substantially improved sample preparation quality but was not sufficient to remove all defect produced during ion beam milling (Fig. 6(b)). A $30 \mathrm{keV} \mathrm{Ga}$ ion beam was used to thin the lamella to the initial thickness of $\sim 165 \mathrm{~nm}$. The calculated projected range of $30 \mathrm{keV} \mathrm{Ga}$ ions in $\mathrm{Al}$ is $24 \mathrm{~nm}$, and considering that TEM lamella is thinned on each side, the total thickness of damaged layer is $\sim 50 \mathrm{~nm}$ [16]. The lamella was then post-processed in PIPS- 2 using $2 \mathrm{keV}, 1 \mathrm{keV}, 500 \mathrm{eV}$, and $300 \mathrm{eV}$ Ar ion beam to the final specimen thickness of $\sim 120 \mathrm{~nm}$. As it can be seen, low energy Ar ion beam milling was not sufficient to completely eliminate the FIB-induced damaged layer, which can be seen as ion beam damage in post-processed $\mathrm{Al}$ specimen. Further low energy milling of the specimen in Al will yield a better quality specimen without sample preparation artifacts. This example was provided to illustrate the importance of projected range in ion beam-based specimen preparation. 
The projected range of both $\mathrm{Ga}$ and $\mathrm{Ar}$ ions in any given material will give operator approximation of the thickness of damaged layer produced during sample preparation, which has to be carefully considered prior to and during milling of the specimens of interest.

\section{Summary}

The key to successful preparation of nuclear materials for TEM without sample preparation artifacts requires a firm understanding of advantages and limitations associated with each sample preparation technique (such as electro-polishing, FIB, and PIPS-2). Electropolishing is a reliable and well-established technique capable of providing reproducible results, which doesn't introduce any defects that can be confused with irradiation-induced damage. Despite the issues intrinsic to highly radioactive specimens and increasing personnel dose at prolonged specimen handling, it has proven to be one of the best options for irradiated nuclear materials. The site-specific sampling nature of the Dual-Beam FIB enables selection and detailed, high spatial resolution characterization of areas of interest, which is critical when analyzing irradiated nuclear fuels where fission product generation creates complex and spatially variant phase constituents. With these FIB advantages come several limitations that must be overcome to obtain high-quality artifact-free TEM specimens from nuclear fuels and materials. The FIB limitations can be ameliorated with low voltage cleaning in PIPS-2 or other comparable systems, which is particularly critical when dealing with ion or neutron irradiated and metallic materials. However, one should always be aware that sample preparation techniques have to be selected carefully for each specimen type and tested on an unirradiated specimen prior to irradiated specimen preparation and analysis.

\section{Acknowledgements}

This work is supported by the U.S. Department of Energy, under DOE Idaho Operations Office Contract DE-AC07-05ID14517. Accordingly, the U.S. Government retains a nonexclusive, royalty-free license to publish or reproduce the published form of this contribution, or allow others to do so, for U.S. Government purposes.

\section{U.S. Department of Energy Disclaimer}

This information was prepared as an account of work sponsored by an agency of the U.S. Government. Neither the U.S. Government nor any agency thereof, nor any of their employees, makes any warranty, express or implied, or assumes any legal liability or responsibility for the accuracy, completeness, or usefulness of any information, apparatus, product, or process disclosed, or represents that is use would not infringe privately owned rights. References herein to any specific commercial product, process, or service by trade name, trademark, manufacturer, or otherwise, does not necessarily constitute or imply its endorsement, recommendation, or favoring by the U.S. Government or any agency thereof. The views and opinions of authors expressed herein do not necessarily state or reflect those of the U.S. Government or any agency thereof. 


\section{References:}

[1] B. L. Eyre, M. J. Sole, J. Nucl. Mater. 18 (1966) 314-322.

[2] A. Pochettino, M. Ipohorski, Ultramicroscopy 3 (1978) 61-67.

[3] J. H. Chute, D. G. Walker, J. Nucl. Mater. 14 (1964) 187-194.

[4] J. L. Brimhall, B. Mastel, J. Nucl. Mater. 29 (1969) 123-125.

[5] A. Munitz, J. Nucl. Mater. 165 (1989) 305-312.

[6] D. V. Sridhara Rao, K. Muraleedharan, C. J. Humphreys, (2011). TEM specimen preparation techniques. In Microscopy: Science, Technology, Applications \& Education, Vol. 2, Mendez- Vilas, A. \& Diaz, J. (Eds.), pp. 1232-1244. Spain: Formatex. ISBN13:978-84-614-6190-5.

[7] J. D. Casey et. al., J. Vac. Sci Technol. B 20 (2002) 2682-2685.

[8] R. Spolenak, L. Sauter, C. Eberl, Scripta Mater. 53 (2005) 1291-1296.

[9] S. Olliges et. al., Acta Mater. 54 (2006) 5393-5399.

[10] B. Miller, J. Gan, J. Madden, J. F. Jue, A. Robinson, D. D. Keiser, J. Nucl. Mater. 424 (2012) 38-42.

[11] D. Tomus, H. P. Ng, Micron 44 (2013) 115-119.

[12] C. M. Hernández-Mayoral, D. Gómez-Briceño, J. Nucl. Mater. 399 (2010) 146-153.

[13] A. Prokhodtseva, B. Decamps, R. Schaublin, J. Nucl. Mater. 442 (2013)5786-5789.

[14] D. Kaoumi, J. Adamson, M. Kirk, J. Nucl. Mater. 445 (2014) 12-19.

[15] D. S. Gelles, J. Nucl. Mater. 108-109 (1982) 515-526.

[16] Ziegler, J. F., Biersack, J. P., Ziegler, M. D., SRIM: the stopping and range of ions in matter, Lulu Press, Morrisville, NC, USA, 2009. 


\section{Caption}

Figure 1. Bright field TEM micrographs of unirradiated HT-9: a) after electro-polishing and b) after preparation using FIB.

Figure 2. Bright field TEM micrographs of pure unirradiated Fe: a) after electro-polishing and b) after preparation using FIB.

Figure 3. Bright field TEM micrographs of Fe: a) unirradiated, b) after neutron irradiation to 0.1 dpa at 300C, and c) after $500 \mathrm{keV} \mathrm{Fe}$ ion irradiation to $0.5 \mathrm{dpa}$ at RT. Figure 3(b) is adapted from Ref. [12] and 3(c) from Ref. [13].

Figure 4. Bright field TEM micrographs of a) unirradiated $\mathrm{Fe}-12 \mathrm{Cr}$, b) $12 \mathrm{Cr}$ model steel irradiated at $200 \mathrm{C}$ with $1 \mathrm{MeV} \mathrm{Kr}$ ions, and c) Fe- $15 \mathrm{Cr}$ neutron irradiated to $2.8 \mathrm{e}^{22} / \mathrm{cm}^{2}$ at $450 \mathrm{C}$. Figure 4(b) is reprinted from Ref. [14] and 4(c) from Ref. [15].

Figure 5. Bright field TEM micrographs of Fe-12Cr: a) after electro-polishing, b) after preparation in FIB, and c) after preparation in FIB and post-processing in PIPS-2.

Table 1. PIPS-2 sample preparation parameters.

Figure 6. Bright field TEM micrographs of $\mathrm{Al}$ : a) after preparation in FIB, and b) after postprocessing in PIPS-2. 


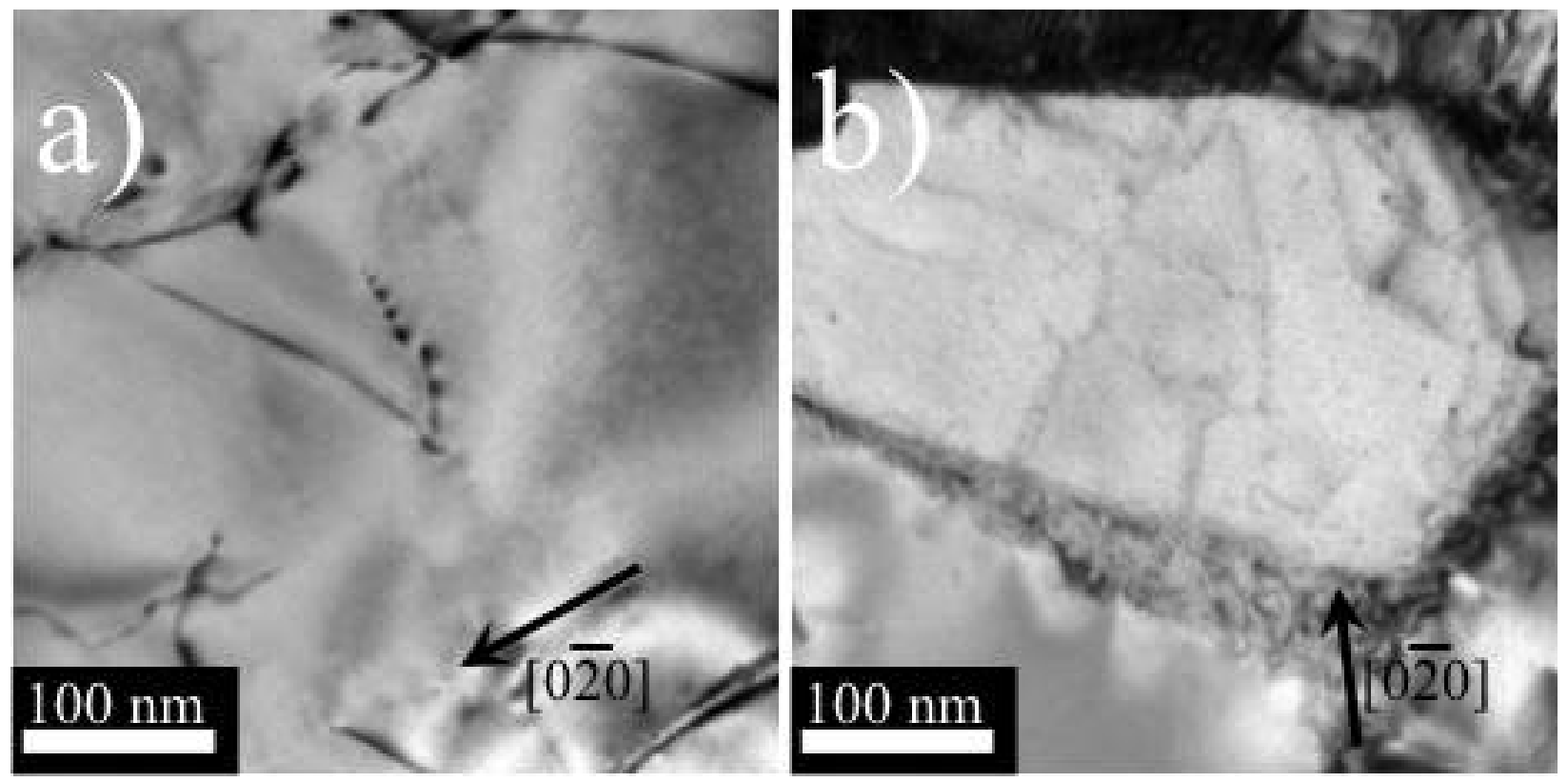

Fig. 1. 


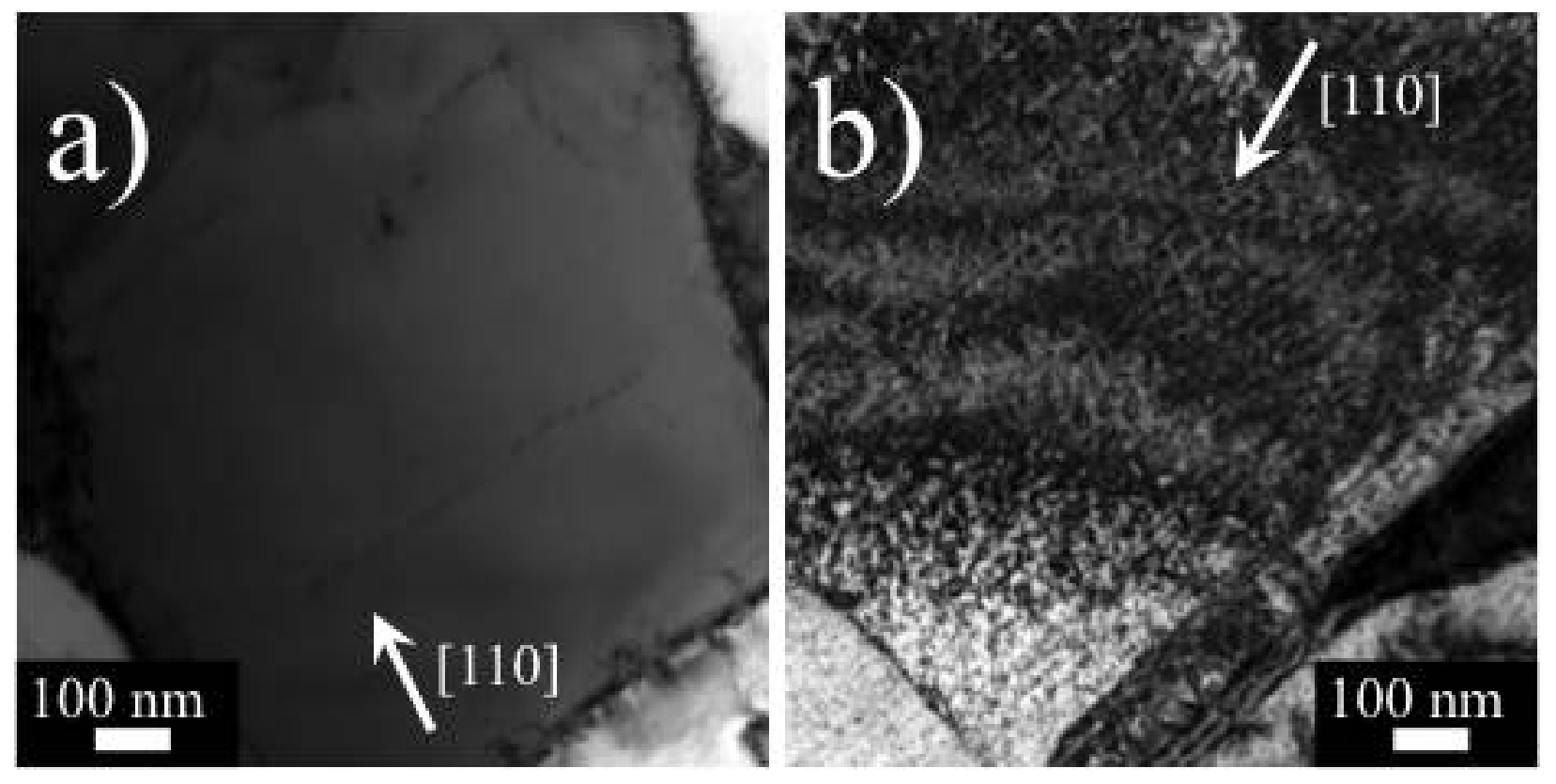

Fig. 2. 

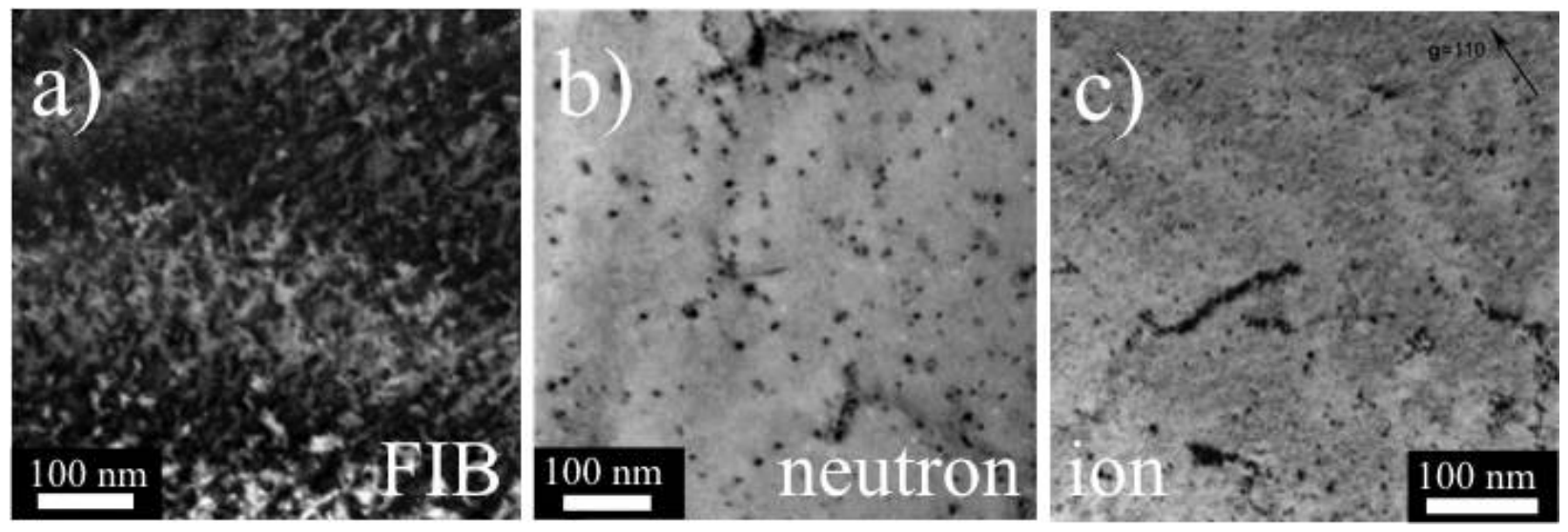

Fig. 3. 

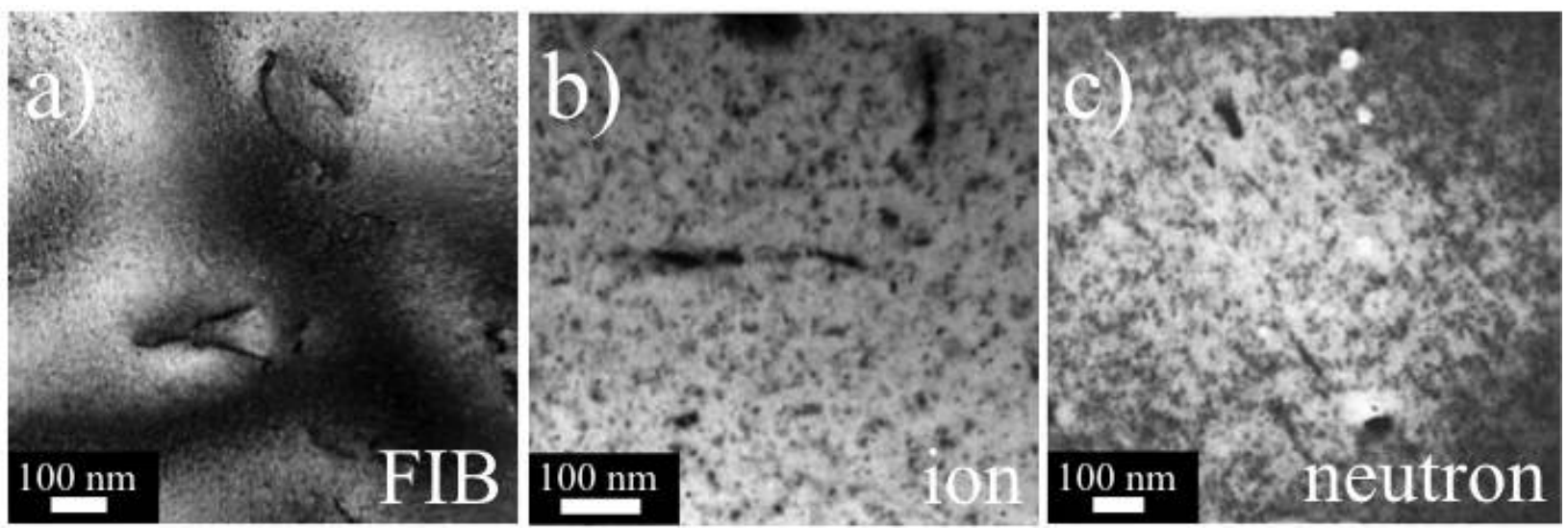

Fig. 4. 


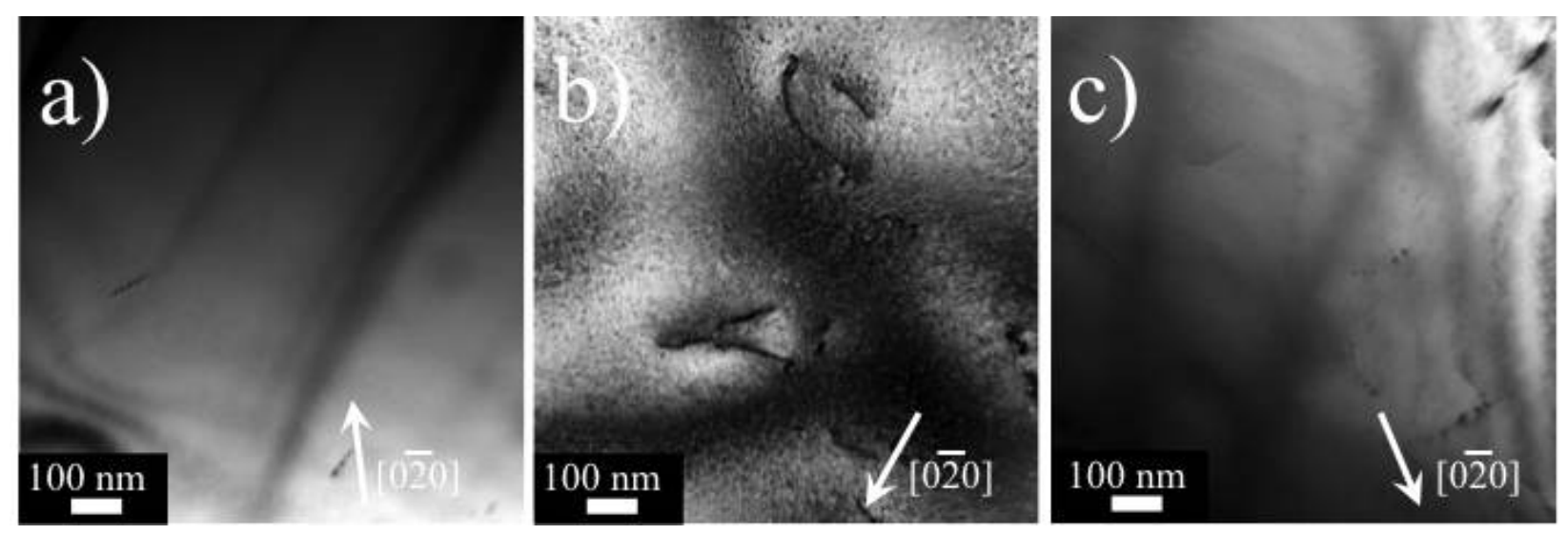

Fig. 5. 


\begin{tabular}{|c|c|c|c|}
\hline Instrument & Energy & Milling angle & Milling temperature \\
\hline PIPS-2 & $2 \mathrm{kV}$ & \pm 8 degrees & RT \\
\hline PIPS-2 & $1 \mathrm{kV}$ & \pm 8 degrees & RT \\
\hline PIPS-2 & $0.5 \mathrm{kV}$ & \pm 8 degrees & RT \\
\hline PIPS-2 & $0.3 \mathrm{kV}$ & \pm 8 degrees & RT \\
\hline
\end{tabular}

Table 1. 

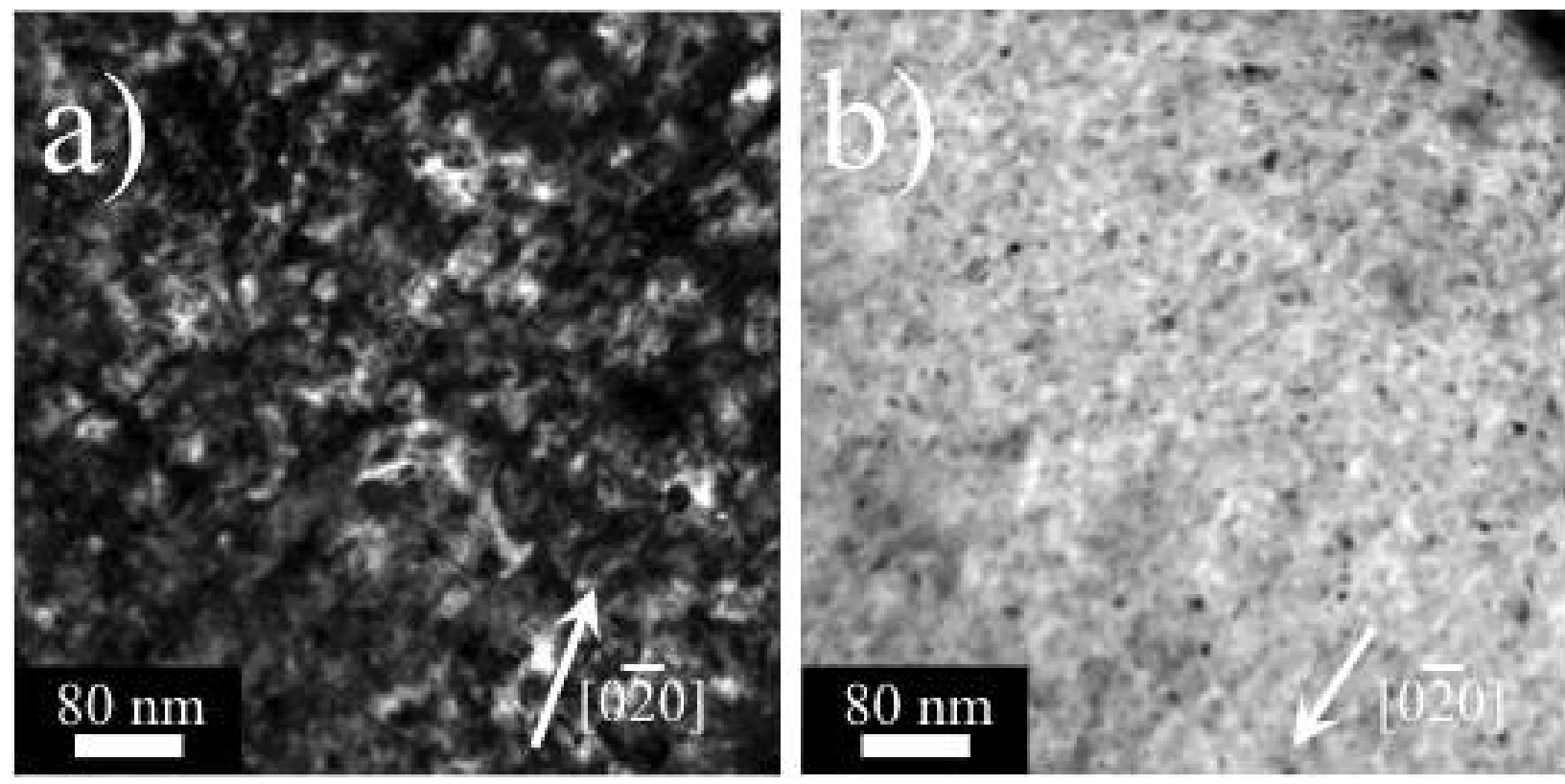

Fig. 6. 